\title{
policy statement
}

\section{Operational Use of Weather Satellites (Adopted by the AMS Council on 18 January 1998)}

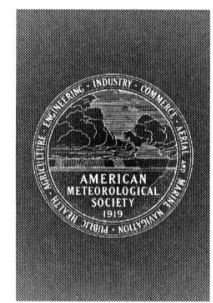

Weather satellites are now a vital component of the world weather and climate monitoring and prediction systems. Therefore, steps must be taken to ensure that their role is both sustained and enhanced to provide a cost effective and efficient satellite system. To this end, the AMS urges the following actions:

- maintain continuity of the current satellite system,

- provide more focus on the calibration of satellite measurements,

- continue coordination of both the national and international satellite systems,

- allow for open access to all satellite data,

- support evolution toward improved sensor systems and acceleration of the transfer of their data into weather forecast services,

- protect remote sensing frequencies,

- emphasize education and training in the optimal use of satellite remote sensing data.

Over the past quarter century advances in satellite technology have been impressive, and weather services throughout the world have become increasingly reliant on meteorological data from satellites. It is now widely accepted that the inclusion of multispectral radiance measurements into numerical models has contributed significantly to the improvement in numerical weather forecasting. In addition, depiction of current weather and short-range forecasting have progressed with the ability to rapidly process and animate high-frequency multispectral imagery available from geostationary satellites and with timely access to microwave and high-resolution multispectral data from polar-orbiting satellites. These animations have provided a reliable and routine method of communicating present and future weather conditions to the public through television broadcasts. Global satellite observations over the last two decades have contributed to a better understanding of climate variability and the

(C)1998 American Meteorological Society effects of natural and man-induced processes on the environment.

Maintenance and improvement of global satellite systems, including a need for accurate ongoing calibration, have become critical necessities for sustaining and enhancing weather and climate prediction services and for monitoring global surface and tropospheric temperature, ozone, and cloud characteristics and associated properties. Our capability must include the global coverage of the polar-orbiting satellites and the continuous, broad coverage of the geostationary satellites. In this coming era of restricted financial resources, it is imperative to maintain and improve the quality of the satellite data and to strengthen the widespread and timely access to the most current information. In addition, it is also critical that civilian, military, and commercial entities in the United States, as well as those in the international remote sensing community, continue to work together to map out the most critical missions to form the basis of an effective, coordinated satellite program.

Many of the advances in both forecasting the local weather and understanding the global climate have occurred because a diverse scientific and user community had open access to global satellite observations. The easy operational access to remote sensing data has ensured against unnecessary data voids, enabled international benefit from the positive impact of the data on the accuracy of weather predictions, encouraged the use of the data by the broad scientific community, and fostered the scientific learning and development of algorithms and services that eventually result in the saving of life and property. Thus it is crucial that access to satellite data remain free and open. Easy access to environmental satellite data will require vigilance, generosity, and vision by scientists and political leaders alike. Continued communications and technology improvements should facilitate this cooperation.

The transfer of new retrieval techniques and sensor technologies from research into operational satellite services remains vitally important. Operational 
centers rely on robust research and development programs to demonstrate useful and reliable science and technology. Moreover, there have not been appropriate resources devoted to the optimal utilization of remote sensing measurements, both before and after deployment. We believe research and development programs are vital to foster new sensor technology, and we must accelerate the incorporation of these new measurements into the weather forecast services by the appropriate government, university, and private sector institutions.

The protection of microwave frequencies for passive remote sensing from space against possible harmful interference from active radio communication services sharing the same bands is essential for safeguarding and further developing environmental sciences, which include operational meteorology, hydrology, and oceanography. The protection of spaceborne remote sensing is obtained through adequate primary frequency allocations that have to be exclusive where needed, or associated with adequate power limits for active radio communication services sharing the bands where possible. We encourage and support international efforts to secure and/or obtain the required primary allocations and protection criteria from World Radiocommunication Conferences of the International Telecommunication Union, which would ultimately protect these important bands for environmental remote sensing.

It is important that the nature and optimal use of satellite remote sensing data be incorporated into the educational curricula for atmospheric sciences at both the college and precollege levels. While both the AMS and the federal government have laid the foundation for promoting meteorological education for both students and science teachers at these levels, significant emphasis on satellite remote sensing needs to be expanded into the primary grades to stimulate greater interest in meteorology at these earlier grade levels.

\section{I3th Conference on Planned and Inadvertent Weather Modification}

\section{January-2 February 1996}

\section{Atlanta, Georgia}

Numerical Cloud Models • Warm Season Cloud Modification •

Hail and Other Severe Storms Modification - Inadvertent

Weather Modification - Global Role of the Private Sector in

Weather Modification Applications • Cold Season/Orographic Cloud Modification

\section{American Meteorological Society}

C1996 American Meteorological Society. Softbound, B\&W, 190 pp., $\$ 60.00$ list $\$ 35.00$ members (includes shipping and handling). Please send prepaid orders to: Order Department, American Meteorological Society, 45 Beacon St., Boston, MA 02108-3693.

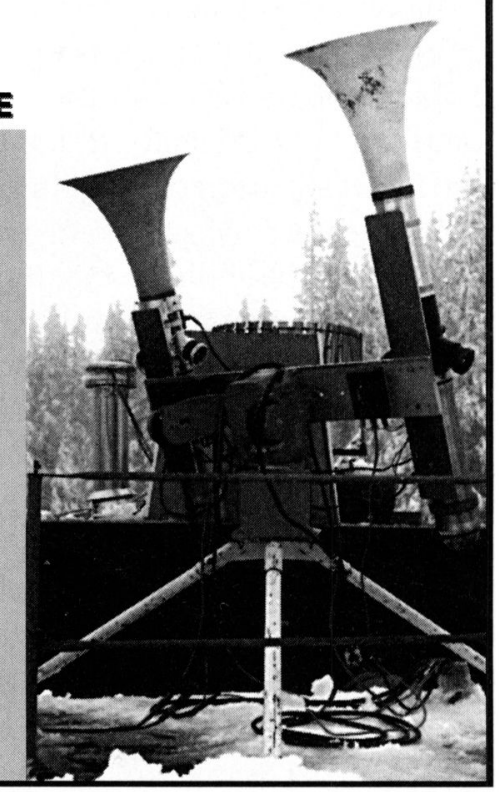

